\title{
Metacognição e as Atividades Experimentais em Ciências: Análise da Produção em Periódicos Estrangeiros
}

\author{
Metacognition and Experimental Activities in Science: Analyzing Articles \\ from Foreign Journals
}

Ana Claudia Tasso dos Santos, ${ }^{(1)}$ Cleci Teresinha Werner da Rosa

Palavras-chave Resumo Neste trabalho é analisado um conjunto de artigos

Atividades estrangeiros que associam a metacognição com as atividades Experimentais; experimentais em Ciências, verificando quais as características

Processos Metacognitivos; presentes nessas pesquisas. Para tanto, desenvolveu-se um estudo junto à base ERIC identificando 19 publicações em língua inglesa.

Revisão em Os resultados evidenciam que as pesquisas em metacognição e Periódicos. atividades experimentais continuam em crescimento e que estão sendo realizadas, com maior frequência, nos Estados Unidos apesar de o continente asiático ter a maior quantidade de países envolvidos em pesquisa nessa área. Além disso, o estudo aponta o componente curricular Química e o ensino de Graduação, como os mais presentes. A organização didático-metodológica verificada na maioria dos artigos foi a atividade experimental investigativa em laboratórios reais, realizada em grupo e sob a orientação de um professor. Em relação ao modo como a metacognição encontra-se associada às atividades experimentais, o estudo revelou que para a maioria das pesquisas a metacognição ocupou lugar de centralidade, todavia, em alguns casos a prática foi modificada para incluí-la e, em outros, ela foi analisada sem a necessidade de alteração. $O$ conceito de metacognição predominante na análise dos resultados dessas investigações foi o relacionado às ações regulatórias do pensamento (autorregulação), utilizadas para planejar, monitorar e avaliar este pensamento e a aprendizagem dos estudantes. 
Keywords Abstract In this study we analyzed a selection of international Experimental publications that associate metacognition and experimental activities Activities; in science, assessing the characteristics of these articles. To this end, we Metacognitive queried the ERIC database, which returned 19 publications in English.

Processes; The results show that research on metacognition and experimental Journal Review. activities continues to grow to be carried out, especially in the U.S., although the Asian continent holds the largest number of countries involved in research in this field. Moreover, we found that chemistry and undergraduate teaching are prevalent terms in the selection. The didactic-methodological organization present in most articles refer to the inquiry-based approach in real laboratories, carried out in groups and under the guidance of a professor. Regarding the way in which metacognition is associated with experimental activities, the study revealed that, for most researchers, metacognition played a central role, however, in some cases, the practice was modified in order to include it, whereas in others it was analyzed without the need for change. The predominant concept of metacognition related to the regulatory actions of thought (self-regulation), which are used to plan, oversee and evaluate this thought and the students' learning.

\section{Introdução}

O modelo de atividades experimentais presente em grande parte dos sistemas de ensino tem sido duramente criticado por pesquisadores como Hodson (1988), PinhoAlves (2000), Borges (2002), Rosa (2011), Heidemann (2015), Marques (2020), entre outros, argumentando que tais atividades pouco contribuem com a apropriação dos conhecimentos por parte dos estudantes, apesar de apresentarem um forte potencial motivacional. Heidemann (2015), por exemplo, frente a tais críticas, assinala a necessidade de que essas atividades estejam fundamentadas em referenciais epistemológicos bem estabelecidos, de modo que não favoreçam a construção de concepções epistemológicas ingênuas por parte dos estudantes.

Autores como Sasseron (2008), Carvalho (2010) e Zômpero e Laburu (2011), entre outros, apontam a abordagem investigativa como possibilidade para (re)significar as atividades experimentais. Nessa abordagem, a atividade toma como ponto de partida um problema genuíno colocado pelo professor e leva o aluno a propor soluções, (criar hipóteses), testá-las e estabelecer conclusões. O uso de situações-problemas e a presença de perguntas abertas, típicos da abordagem investigativa, trazem um conjunto de aspectos que possibilitam um movimento cognitivo nos estudantes que se diferencia do provocado pelo modelo dos roteiros rígidos. Próximo a essa abordagem, temos o denominado "Ensino por Indagação", proposto por Meneses Villagrá (2018), que se revela promissor, no seu entendimento, por instigar nos estudantes a busca por respostas a situações-problemas. 
O autor associa essa abordagem às atividades experimentais e mostra a sua relevância em termos de oportunizar aos estudantes a formulação de perguntas e seu posterior refinamento, formulação de hipóteses e discussões sobre elas, bem como as conclusões estabelecidas a partir das atividades desenvolvidas.

Nesse contexto de críticas e alternativas direcionadas às atividades experimentais, Rosa (2011) mostra que essas atividades desenvolvidas a partir dos roteiros rígidos pouco favorecem o processo de reflexão dos alunos sobre a forma como aprendem e os mecanismos cognitivos que põem em movimento para aprender. A autora infere que tais atividades deveriam ser estruturadas de forma a privilegiar a tomada de consciência dos estudantes sobre seus conhecimentos (ou a falta deles) e sobre as características da tarefa a ser realizada. Além disso, ela aponta a necessidade de os alunos aprenderem a autorregular suas ações, recorrendo a um planejamento, monitorando e avaliando o que realizaram durante a atividade. Tais processos, entendidos como metacognitivos, levam a autora a ponderar que as atividades experimentais organizadas por roteiros rígidos com resultados antecipados pelos professores, pecam em termos de favorecer a evocação dessa forma de pensamento de cunho reflexivo.

Na mesma linha, autores como Hattie (2012) e Fadel et al., (2015), entre outros, mostram que a identificação dos próprios conhecimentos e a habilidade de regular e controlar o pensamento, entendidas por eles como metacognição, se revelam essenciais na qualificação da aprendizagem. Tal inferência leva em consideração que ao tomar consciência de seus próprios conhecimentos e controlar suas ações, os sujeitos se tornam mais bem-sucedidos na busca por atingir seus objetivos. A reflexão do pensamento promove o questionamento que, conforme Santos (2008), decorre da liberdade de pensamento. De acordo com o autor, os estudantes precisam voltar-se "às coisas simples, à capacidade de formular perguntas simples, perguntas que, como Einstein costumava dizer, só uma criança pode fazer mas que, depois de feitas, são capazes de trazer uma luz nova à nossa perplexidade" (p. 15).

Malone (2008) e Taasoobshirazi e Farley (2013), partidários dessa compreensão, inferem que os alunos que evocam tal pensamento acabam atingindo de forma mais satisfatória o êxito na atividade. Taasoobshirazi e Farley (2013), por exemplo, mencionam que estudantes considerados com expertise em resolução de problemas em Física recorrem mais intensamente a seus recursos metacognitivos, quando comparados com estudantes que apresentam dificuldades nesse tipo de tarefa. No estudo, os autores destacam, entre outras variáveis, que os experts utilizam estratégias metacognitivas mais apuradas e tendem a responder corretamente os problemas, enquanto aqueles que usam uma estratégia mais simples tendem a respondê-los incorretamente.

Todavia, precisamos considerar que os estudos envolvendo o uso da metacognição associado às estratégias didáticas têm encontrado problemas, dentre os quais a própria definição do termo, uma vez que este vem sendo utilizado em diferentes campos que procedem a detalhamentos e aproximações peculiares às suas áreas, como apontam Zohar e Barzilai (2013). No entanto, seguem as autoras, as pesquisas vinculadas ao campo 
da Educação em Ciências têm adotado um entendimento que aproxima a metacognição da definição dada por Flavell - considerado o precursor nos estudos em metacognição - em 1979 e ampliada na obra publicada em 1999. A compreensão do autor envolve o conhecimento que o sujeito tem sobre seus próprios conhecimentos e a capacidade dada aos processos de regulação do controle executivo. Nesse entendimento, incluemse as experiências metacognitivas, o conhecimento metacognitivo e as habilidades metacognitivas. Hacker (1998) expressa essas componentes mostrando que elas estão interligadas e constituem o pensamento metacognitivo. Ainda, conforme o autor, esse pensamento está relacionado àquilo que alguém sabe - conhecimento cognitivo do que está atualmente realizando - habilidade metacognitiva-, e do estado afetivo ou cognitivo atual de alguém - experiência metacognitiva.

Na operacionalização em contexto educativo, encontramos que a compreensão da metacognição tem sido assinalada desde duas componentes e seus respectivos elementos metacognitivos: conhecimento do conhecimento (conhecimento metacognitivo), envolvendo as variáveis pessoa, tarefa e estratégia; controle executivo e autorregulador (habilidades metacognitivas), relacionados a planejamento, monitoramento e avaliação. Essa compreensão, que traz as experiências metacognitivas como aspecto que perpassa as duas componentes citadas, tem subsidiado pesquisas direcionadas a qualificar o processo de aprendizagem em Ciências. Todavia, essas ações didáticas, como assinalam Zohar e Barzilai (2013), variam especialmente em termos da presença dos componentes e elementos metacognitivos. Essa identificação foi apontada em estudos como o desenvolvido por Rosa e Meneses Villagrá (2018), que, embora não limitado às atividades experimentais, mostrou que a operacionalização da metacognição em situações didáticas em Física tem sido restrita à sua compreensão como reflexão, ou, alternativamente, quando há uma ampliação desse entendimento, restringe-se a componente vinculado ao controle executivo e autorregulador - habilidades metacognitivas (planejamento, monitoramento e avaliação).

Essa compreensão da metacognição abordada nos estudos pode ser contrastada com outras intervenções, como a de Kipnis e Hofstein (2008) e de Rosa (2011), em que há o incremento explícito da componente vinculada à tomada de consciência dos sujeitos sobre seus próprios conhecimentos (conhecimento do conhecimento) e seus elementos (pessoa, tarefa e estratégia). Tais estudos nos levam a buscar, na literatura estrangeira - recorte do estudo, as pesquisas que abordam a metacognição como coadjuvante das atividades experimentais, com vistas a entender que abordagem de atividades experimentais tem sido utilizada pelos pesquisadores e como a metacognição é compreendida e associada a esses estudos.

Portanto, imbuídas do desejo de repensar as atividades presentes no ensino de Ciências, partimos para a verificação junto à literatura especializada, particularmente artigos estrangeiros, do modo como a metacognição tem sido considerada nos estudos vinculados às atividades experimentais no ensino de Ciências - Física, Química e Biologia. Para tanto, tomamos como questionamento central a seguinte indagação: quais 
características são identificadas nas pesquisas estrangeiras que vinculam a metacognição com as atividades experimentais no ensino de Ciências? Por características definimos aspectos relacionados a um panorama geral das pesquisas, para identificar a distribuição dos artigos (ano, periódico, local geográfico, nível de ensino e componente curricular envolvido), bem como a análise frente ao lugar da metacognição nessas atividades (ambiente de aprendizagem, organização didático-metodológica presente nas atividades experimentais e entendimento de metacognição). Diante desses aspectos, temos como objetivo principal realizar uma revisão de artigos no campo da Educação em Ciências que associam as atividades experimentais aos processos metacognitivos.

Frente ao exposto nos ocupamos de desenvolver uma pesquisa bibliográfica de modo a trazer o cenário dos estudos que relacionam as atividades experimentais com a metacognição.

\section{Metodologia}

No desenvolvimento de uma pesquisa, o investigador deve escolher uma abordagem metodológica que varia de acordo com as características do conhecimento que deseja produzir. Dentre as possibilidades de metodologias, está a abordagem qualitativa, selecionada para o presente estudo. Ela está centrada no aprofundamento da compreensão das relações entre o sujeito e o mundo, cujos aspectos não podem ou não deveriam ser expressos em números (Gil, 1994), porque engloba o "universo dos significados, dos motivos, das aspirações, das crenças, dos valores e das atitudes" (Deslandes et al., 2012, p. 21).

Além da abordagem qualitativa o estudo encontra-se vinculado a uma pesquisa bibliográfica como expressa por Gil (1994). Segundo o autor esse tipo de pesquisa utiliza, como fonte de consulta, investigações acadêmicas publicadas em uma determinada base de dados o que leva a identificá-la com o que Romanowski (2002) denomina de "estado do conhecimento". O objetivo dessas pesquisas consiste em reconhecer "o que já se sabe, as principais lacunas, onde se encontram os principais entraves teóricos e/ ou metodológicos" (Luna, 2011, pp. 87-88). Segue o autor mencionando que esses estudos são fontes importantes para atualização dos profissionais ao possibilitar o acompanhamento do desenvolvimento, das transformações e das inovações na área.

Para tanto, e como forma de estabelecer o corpus, tomamos como ponto de partida o estudo desenvolvido por Rosa, Darroz e Rosa (2017). Após analisarem publicações em 14 periódicos nacionais (2007-2016), esses autores identificaram que, aproximadamente, $0,13 \%$ de um universo de 3.849 investigações, associava a metacognição às atividades experimentais no ensino de Física. A escassez desses estudos na literatura nacional confere a essa pesquisa o enfoque em investigações estrangeiras, com o intuito de avaliar como vem sendo realizada a associação entre a metacognição e as atividades experimentais.

Nesse contexto de busca pela literatura estrangeira, identificamos na base de dados Education Resources Information Center (ERIC) a possibilidade de determinação do corpus de análise. Essa base de dados tem servido de referência para outros estudos que realizaram análises de artigos como o realizado por Ferreira (2012), Zohar e Barzilai 
(2013), Bomfim et al., (2016) e Rosa e Meneses Villagrá (2018). Destacamos, ainda, que a ERIC engloba os periódicos mais expressivos no campo da Educação em Ciências, bem como o Metacognition and Learning, considerado o mais especializado na área de aprendizagem envolvendo processos metacognitivos.

Como descritores utilizamos as expressões: experimental activities, laboratory, laboratory experiences, metacognition, metacognitive, combinadas de seis formas diferentes: "experimental activities AND metacognition"; "experimental activities AND metacognitive"; "laboratory AND metacognition"; "laboratory AND metacognitive"; "laboratory experiences AND metacognition"; "laboratory experiences AND metacognitive". Como recorte temporal definimos as pesquisas desenvolvidas após o ano 2000, pois, segundo Zohar e Barzilai (2013), é a partir desse período que há um crescente interesse da comunidade acadêmica pela metacognição.

Após a submissão das seis combinações suprarreferidas, realizamos o agrupamento dos resultados, totalizando 554, entre artigos, teses e dissertações. O primeiro parâmetro estabelecido para triagem dos documentos foi a eliminação das publicações repetidas, resultando em 356 textos não repetidos. Na sequência, procedemos à leitura do título, do resumo e das palavras-chave, tendo como segundo critério de seleção da amostra encontrar os seguintes termos: "experimental activities", "laboratory", "laboratory experiences", "metacognition", "metacognitive", "science”, "physics", "chemistry" e "biology". Após esse filtro, restaram 50 artigos, que se reduziram a 19, após uma leitura atenta a seus resumos, eliminando aqueles que utilizavam a palavra "metacognition" sem estabelecer uma relação direta com as atividades experimentais mencionadas no estudo. Dessa forma, o corpus ficou restrito aos 19 artigos que serão analisados na próxima seção e que seguem identificados na Figura 1, onde apresentamos os respectivos títulos, autores, anos de publicação e periódicos.

Figura 1. Relação dos artigos selecionados como objeto de estudo

\begin{tabular}{|l|l|}
\hline $\begin{array}{c}\text { Ordem dos } \\
\text { artigos }\end{array}$ & \multicolumn{1}{c|}{ Títulos, autores, anos de publicação e periódicos } \\
\hline T1 & $\begin{array}{l}\text { Clark, R. L., Clough, M. P., Berg, C. A. (2000). Modifying Cookbook Labs to Mentally } \\
\text { Engage Students. The Science Teacher, 67(7), 40-43. }\end{array}$ \\
\hline T2 & $\begin{array}{l}\text { Davidowitz, B., \& Rollnick, M. (2003). Enabling metacognition in the laboratory: a } \\
\text { case study of four second year University Chemistry Students. Research in Science } \\
\text { Education, 33(1), 43-69. }\end{array}$ \\
\hline T3 & $\begin{array}{l}\text { Hand, B., Wallace, C. W., \& Yang, E-M. (2004). Using a Science Writing Heuristic } \\
\text { to enhance learning outcomes from laboratory activities in seventh-grade science: } \\
\text { quantitative and qualitative aspects. International Journal of Science Education, 26(2), } \\
\text { 131-149. }\end{array}$ \\
\hline T4 & $\begin{array}{l}\text { Carillo; L., Lee, C., \& Rickey, D. (2005). Enhancing Science Teaching by Doing More: } \\
\text { A Framework to Guide Chemistry Students' Thinking in the Laboratory. The Science } \\
\text { Teacher, 72(7), 60-64. }\end{array}$ \\
\hline T5 & $\begin{array}{l}\text { Kung, R. L., \& Linder, C. (2007). Metacognitive activity in the physics student } \\
\text { laboratory: is increased metacognition necessarily better? Metacognition Learning, } \\
\text { 2(1), 41-56. }\end{array}$ \\
\hline
\end{tabular}

Fonte: autoria própria. 
Figura 1. Relação dos artigos selecionados como objeto de estudo (continuação)

\begin{tabular}{|c|c|}
\hline $\begin{array}{l}\text { Ordem dos } \\
\text { artigos }\end{array}$ & Títulos, autores, anos de publicação e periódicos \\
\hline T6 & $\begin{array}{l}\text { Kipnis, M., \& Hofstein, A. (2008). The inquiry laboratory as a source for development } \\
\text { of metacognitive skills. International Journal of Science and Mathematics Education, } \\
6(3), 601-627 .\end{array}$ \\
\hline T7 & $\begin{array}{l}\text { Sandi-Urena, S., Cooper, M. M., \& Gatlin, T. A. (2011). Graduate teaching assistants' } \\
\text { epistemological and metacognitive development. Chemistry Education Research and } \\
\text { Practice, 12(1), 92-100. }\end{array}$ \\
\hline $\mathrm{T} 8$ & $\begin{array}{l}\text { Sandi-Urena, S., Cooper, M. M., Gatlin, T. A., \& Bhattacharyya, G. (2011). Students' } \\
\text { experience in a general chemistry cooperative problem based laboratory. Chemistry } \\
\text { Education Research and Practice, 12(4), 434-442. }\end{array}$ \\
\hline T9 & $\begin{array}{l}\text { Sandi-Urena, S., Cooper, M. M., Stevens, R. (2012). Effect of Cooperative Problem- } \\
\text { Based Lab Instruction on Metacognition and Problem-Solving Skills. Journal of } \\
\text { Chemical Education, 89(6), 700-706. }\end{array}$ \\
\hline T10 & $\begin{array}{l}\text { Llorens-Molina, J. A., Jaime, J. M. L., \& Berzosa, I. S. (2012). Analysis of students' } \\
\text { generated questions in laboratory learning environments. Journal of Technology and } \\
\text { Science Education, 2(1), 46-55. }\end{array}$ \\
\hline T11 & $\begin{array}{l}\text { Saribaş, D., Mugaloğlu, E. Z., \& Bayram, H. (2013). Creating metacognitive awareness } \\
\text { in the lab: outcomes for preservice science teachers. Eurasia Journal of Mathematics, } \\
\text { Science \& Technology Education, 9(1), 83-88. }\end{array}$ \\
\hline T12 & $\begin{array}{l}\text { Çalişkan, I. (2014). The Perceptions of Pre-Service Science Teachers` about Using } \\
\text { Vee Diagrams and Electronic Portfolios in Physics Laboratuary Course. Education } \\
\text { Research and Reviews, 9(6), 173-182. }\end{array}$ \\
\hline T13 & $\begin{array}{l}\text { Bagán, H., Sayós, R., \& García, J. F. (2015). Skill development in experimental courses. } \\
\text { Journal of Technology and Science Education, 5(3), 169-183. }\end{array}$ \\
\hline T14 & $\begin{array}{l}\text { Van Optsal, M. T., \& Daubenmire, P. L. (2015). Extending Students' Practice of } \\
\text { Metacognitive Regulation Skills with the Science Writing Heuristic. International } \\
\text { Journal of Science Education, 37(7), 1089-1112. }\end{array}$ \\
\hline T15 & $\begin{array}{l}\text { Bruckermann, T., Aschermann, E., Bresges, A., \& Schlüter, K. (2017). Metacognitive } \\
\text { and multimedia support of experiments in inquiry learning for science teacher } \\
\text { preparation. International Journal of Science Education, 39(6), 701-722. }\end{array}$ \\
\hline T16 & $\begin{array}{l}\text { Mathabathe, K. C., \& Potgieter, M. (2017). Manifestations of metacognitive activity } \\
\text { during the collaborative planning of chemistry practical investigations. International } \\
\text { Journal of Science Education, 39(11), 1465-1484. }\end{array}$ \\
\hline T17 & $\begin{array}{l}\text { Ghani, I. B. A., Ibrahim, N. H., Yahaya, N., Surif, J. (2017). Enhancing students' } \\
\text { HOTS in Laboratory Educational Activity by using Concept Map as an Alternative } \\
\text { Assessment Tool. Chemistry Education Research and Practice, 18(4), 949-874. }\end{array}$ \\
\hline T18 & $\begin{array}{l}\text { Teichert, M. A., Tien, L. T., Dysleski, L., \& Rickey, D. (2017). Thinking Processes } \\
\text { Associated with Undergraduate Chemistry Students' Success at Applying a Molecular- } \\
\text { Level Model in a New Context. Journal of Chemical Education, 94(9), 1195-1208. }\end{array}$ \\
\hline T19 & $\begin{array}{l}\text { Achuthan, K., Francis, S. P., \& Diwakar, S. (2017). Augmented reflective learning } \\
\text { and knowledge retention perceived among students in classrooms involving virtual } \\
\text { laboratories. Education and Information Technologies, 22(6), 2825-2855. }\end{array}$ \\
\hline
\end{tabular}

Fonte: autoria própria. 


\section{Resultados e Discussões}

A partir da seleção dos artigos apresentados na Figura 1, passamos à etapa de discussões, buscando identificar as características dessa produção. Tais características foram entendidas e delimitadas como sendo o panorama geral das pesquisas e a metacognição presente nos artigos estrangeiros. No caso da categoria "Panorama geral das pesquisas", identificamos seus elementos como aqueles que permitem analisar de forma mais geral aspectos vinculados à produção da pesquisa. No que tange à categoria "A metacognição presente nas pesquisas", procedemos a uma adaptação em categorias anunciadas no estudo de Zohar e Barzilai (2013), de modo a ajustá-la ao objetivo de nossa investigação. Assim, ressaltamos que o foco esteve em analisar o cenário da produção e identificar como a metacognição se revela presente nas atividades experimentais, identificando, nesse último caso, como estão arranjados esses dois temas. A seguir, nos ocupamos de descrever e analisar os aspectos mencionados e integrantes da análise e da discussão dos dados, iniciando pelo panorama geral das pesquisas e apresentando, logo após, as especificidades dos elementos do estudo, quais sejam: atividades experimentais e metacognição.

\section{Panorama geral das pesquisas}

A presente categoria reúne as características gerais das pesquisas, dentre as quais estão: distribuição dos artigos por ano; periódicos; distribuição geográfica; nível de ensino; e campo do conhecimento ou componente curricular envolvido. Para apresentação e discussão dos dados nessa categoria optamos por apresentar os dados associados a cada item investigados e, ao final, expor as discussões sobre o que foi encontrado no estudo.

Como pode ser visualizado na Figura 1, a distribuição dos artigos em cada um dos dezoito anos que compõem o recorte temporal, revelou que foram publicados: um artigo por ano em 2000, 2003, 2004, 2005, 2007, 2008, 2013 e 2014; dois artigos nos anos de 2011, 2012 e 2015; e, cinco artigos em 2017. Desse conjunto de dados percebemos que a diluição das investigações em doze dos dezoito anos investigados pode ser um indicativo da importância da temática e seu caráter não efêmero. Outro dado que chama a atenção é que nos últimos nove anos (2009-2017) foi divulgado o dobro de investigações em relação aos primeiros nove anos (2000-2008). Disso inferimos que os estudos desenvolvidos sobre essa temática vêm se expandindo ao longo do tempo, a exemplo do mencionado por Zohar e Barzilai (2013). Uma das tendências apontadas pelas autoras é a rápida ampliação do número de pesquisas indexadas na base de dados ERIC que associam a metacognição ao ensino de Ciências. Esse crescimento se torna especialmente notável na comparação de dois períodos: 1990-2002 versus 2000-2012. Uma pesquisa realizada com os mesmos descritores produziu 57 resultados para o primeiro intervalo enquanto que no segundo período foram identificados 233 estudos, indicando um aumento de 308,77\%. 
Desses 233 resultados, 178 artigos atenderam aos critérios de inclusão definidos por Zohar e Barzilai (2013) para a constituição do corpus da pesquisa. As expressões "laboratório" e "experimentos" estiveram presentes no resumo de 12,36\% e 7,30\% do corpus, respectivamente. Para as autoras, as atividades experimentais hands-on são recorrentes na associação entre a metacognição e o ensino de Ciências. A partir da leitura do artigo, não é possível evidenciar se houve estudos que mencionaram ambos os termos.

No quesito periódico, é possível verificar na Figura 1 que nove periódicos estrangeiros foram responsáveis pela publicação dos artigos. Dentre eles, o que teve o maior número de investigações foi o International Journal of Science Education, com quatro artigos. Na sequência, identificamos o Chemistry Education Research and Practice com três artigos publicados. Tomando como parâmetro a pesquisa relatada no parágrafo anterior e desenvolvida por Zohar e Barzilai (2013), identificamos uma diferença em relação ao periódico com maior número de publicações que, no caso do estudo dessas autoras, foi o Chemistry Education Research and Practice.

Em termos da distribuição geográfica, identificamos que os pesquisadores têm realizado investigações sobre a metacognição associada às atividades experimentais em oito países, a saber: África do Sul (T2; T16); Estados Unidos (T1; T3; T4; T5; T7; T8; T9; T14; T18), Índia (T19), Israel (T6), Malásia (T17), Turquia (T11; T12), Alemanha (T15) e Espanha (T10; T13).

Os dados apresentados revelam que os pesquisadores estadunidenses vêm demonstrando maior interesse nessa área do que os pesquisadores de outros países, pelo menos considerando o recorte do presente estudo. Conjecturamos que esse fato pode ter duas explicações, sendo a primeira relativa à língua vernácula dos norte-americanos que favorece a publicação em revistas que são indexadas na base de dados ERIC. A segunda considera que essa temática tem despertado maior interesse em pesquisadores nesse país do que em outros.

A predominância de estudos norte-americanos também é destacada por Rosa e Meneses Villagrá (2018) ao realizarem um estado do conhecimento sobre as intervenções didáticas em Física guiadas pela metacognição. Os autores afirmam que "o tema não está associado a centros de investigação específicos, ainda que existam focos de estudos na temática, especialmente nos Estados Unidos" (p. 592).

A Ásia, o maior continente tanto em território quanto em população, também é o líder no ranking de quantidade de países envolvidos em pesquisa nessa área, totalizando quatro países em comparação a três europeus, um africano e um americano. A contribuição dos pesquisadores asiáticos para o desenvolvimento da temática não pode ser menosprezada uma vez que constitui entre um quarto e um sexto do total de estudos identificados. Em razão da natureza transcontinental da Turquia, para montar o ranking de países por continente, consideramos mais adequado contabilizá-la tanto para o continente asiático quanto para o europeu. 
A quarta característica enquadrada nessa primeira categoria do estudo é a classificação do nível ou grau de ensino, que foi realizada para 16 dos 19 trabalhos, uma vez que em três deles não estava expresso o nível de escolarização em que o estudo foi desenvolvido. Para a distribuição, procedemos à adaptação ao nível de escolarização equivalente no sistema educacional brasileiro, com o objetivo de facilitar a visualização por parte do leitor. Tal distribuição ficou assim identificada nos 16 estudos: Ensino Fundamental - Anos Finais, com um estudo (T3); Ensino Médio, com um estudo (T6); Ensino Superior - Graduação, com 13 estudos (T2; T5; T8; T9; T10; T11; T12; T13; T14; T15; T16; T18; T19); e Pós-Graduação, com um estudo (T7).

A escassez de estudos com os jovens estudantes também chamou a atenção na pesquisa desenvolvida, principalmente com os que frequentam o Ensino Fundamental - Anos Iniciais, contrastada com o grande número de pesquisas conduzidas com alunos do Ensino Superior, que correspondem a 13 estudos. Tal situação já havia sido constatada em nível mais moderado por Zohar e Barzilai (2013) com cerca de 30\% da amostra investigada pelas autoras e de forma mais acentuada por Rosa e Meneses Villagrá (2018), com 50\% do total de artigos analisados. Porém, o interesse por esse nível escolar nem sempre foi tão expressivo. No caso do trabalho de Davidowitz e Rollnick (2003, p. 45), o ensino de Graduação como foco do estudo foi justificado pelo fato de que essa área estava recebendo pouca atenção na literatura. Essa declaração pode explicar por que esse nível de ensino passou a receber um crescente número de investigações a partir dos anos 2000.

Em relação à ausência no Ensino Fundamental - Anos Iniciais, recorremos ao exposto por Galiazzi et al. (2001), que, embora se refiram ao contexto nacional, apontam que nessa etapa da escolarização há pouca atividade experimental. Além disso, há uma escassez de estudos associando a metacognição ao ensino de Ciências nesse nível de ensino. Sobre isso, Zohar e Barzilai (2013, p. 26, tradução nossa) afirmam que em relação aos seus achados na pesquisa

o número de estudos foi proporcional à idade dos estudantes, havia um número muito pequeno de estudos conduzidos com crianças em idade pré-escolar que ia aumentando gradualmente com a idade. Consequentemente, a quantidade de estudos sobre metacognição e ensino de ciências entre jovens aprendizes na préescola e no Ensino Fundamental como um todo é insuficiente. (Zohar; Barzilai, 2013, p. 26, tradução nossa).

Vale ressaltar que os artigos T1, T4 e T17 não mencionam o nível de ensino em que os participantes estavam matriculados, porém as discussões no texto apontam que os estudantes envolvidos estavam vinculados à Educação Básica. O artigo T4 relatou que a pesquisa foi realizada em uma escola secundária, que é responsável pela educação fornecida nos graus que correspondem ao Ensino Fundamental - Anos Finais e ao Ensino Médio. As temáticas escolhidas para a realização das atividades experimentais no artigo desenvolvido por Clark, Clough e Berg (2000) - T1 vinculado à Lei de Conservação da Massa, e no artigo desenvolvido por Ghani et al. (2017) - T17 sobre Eletrólise, sugerem que essas investigações foram conduzidas no Ensino Fundamental - Anos Finais ou no Ensino Médio. 
A quinta característica diz respeito ao campo do conhecimento ou componente curricular em que foi conduzida a atividade experimental associada à metacognição. Como resultado foi identificado que 14 estudos estavam associados à Química (T1; T2; T4; T6; T7; T8; T9; T10; T11; T13; T14; T16; T17; T18), três à Física (T5; T12; T19) e dois à Biologia (T3; T15). A Química foi o componente curricular que teve o maior número de pesquisas, revelando-se o foco das investigações no campo das atividades experimentais associadas à metacognição, como será discutido mais adiante. No estudo de Zohar e Barzilai (2013), encontramos resultados distintos para os 178 estudos analisados: a Biologia foi o campo do conhecimento mais estudado (23\%), seguido da Física $(19,1 \%)$ e da Química (15,2\%). Todavia, o estudo de Zohar e Barzilai (2013) não contemplava estritamente as atividades experimentais.

Se excluído o recorte "atividades experimentais", o campo do conhecimento com o maior número de investigações dentro da Educação em Ciências pela base ERIC, tem sido a Biologia com 166 resultados. Na sequência, temos a Química e a Física, com 133 e 120 retornos, respectivamente. No confronto dos dados, percebemos que a Biologia se revela com um número expressivo de estudos envolvendo metacognição, contudo, reduzido quando se trata do recorte vinculado às atividades experimentais.

Uma questão que pode ser observada é que todos os artigos conduzidos na África do Sul e na Espanha foram realizados no componente curricular Química com alunos da Graduação. Isso pode indicar que esses pesquisadores demonstram um interesse maior por essa disciplina e esse nível escolar. Outra observação importante é que a disciplina de Química foi a que teve o contexto mais vasto de aplicação, abrangendo os quatro níveis escolares nos quais foram realizadas as pesquisas. Em segundo lugar se encontra a Biologia, que foi associada tanto ao Ensino Fundamental quanto à Graduação. Por último, a Física, que somente foi estudada junto aos estudantes universitários.

A partir da análise feita nessa categoria referente ao panorama das 19 pesquisas selecionadas para o estudo, prosseguimos na busca por especificar o modo como a metacognição esteve presente nas atividades experimentais, o que nos ocupamos de relatar e discutir na próxima seção.

\section{A metacognição e as atividades experimentais presentes nos artigos estrangeiros}

Essa categoria agrupa aspectos relacionados à metacognição e às atividades experimentais presentes nos artigos investigados. Especificamente buscamos abordar o lugar da metacognição nas atividades experimentais, o ambiente de aprendizagem, a organização didático-metodológica seguida nas atividades experimentais e $\mathrm{o}$ entendimento de metacognição evidenciada nos estudos.

O primeiro aspecto diz respeito ao lugar da metacognição nas atividades experimentais. Com base na leitura dos artigos, constatamos que em 13 estudos (T2; T3; T5; T6; T7; T8; T9; T11; T14; T15; T16; T18; T19) ela ocupava lugar central e em seis, lugar marginal (T1; T4; T10; T12; T13; T17). Tal classificação tomou por referência o estudo de Zohar e Barzilai (2013), ao mencionarem que central se refere ao 
entendimento de que a metacognição está explicitada nos objetivos da pesquisa, ou seja, o estudo declaradamente menciona que o objetivo está em oportunizar a ativação do pensamento metacognitivo. Por marginal as autoras inferem o estudo que, embora não tenha declaradamente o objetivo de promover a metacognição, acaba apontando para ela nos resultados, como algo associado à atividade.

Interpretamos como positiva a opção predominante por estudos na perspectiva de uma metacognição central, pois nesse caso a sua integração na investigação é mais explicita e pode favorecer de maneira mais abrangente a ativação dessa forma de pensamento. Como exemplo mencionamos o estudo de Kipnis e Hofstein (2008) - T6 cujo objetivo estava em analisar a influência das atividades investigativas no aprimoramento das habilidades metacognitivas. No caso do trabalho desenvolvido por Carillo et al., (2005) - T4, o lugar da metacognição é marginal, porque o termo é citado na introdução, ao mencionar o benefício daquela atividade segundo um estudo anterior de um dos autores. Além disso, sutilmente aparece nos resultados, ao se afirmar que as atividades experimentais utilizadas no estudo colaboram para que os alunos aprendam a aprender.

O segundo aspecto que investigamos nessa categoria está relacionado às pesquisas que destinam à metacognição lugar de centralidade e consideram sua relação com o ambiente em que foram desenvolvidas. Para tanto, e a exemplo da classificação anterior, com base nas discussões de Zohar e Barzilai (2013) e na leitura dos 13 artigos, procedemos à classificação segundo o proposto pelas autoras: ambiente natural; ambiente orientado para metacognição; e ambiente orientado pela metacognição. Como ambiente natural foram enquadrados aqueles estudos em que a prática do professor não é alterada (T5; T6; T7; T8; T9). Como ambiente orientado para metacognição designamos aqueles em que a prática sofre modificação para contemplar a ativação do pensamento metacognitivo (T2; T3; T14; T16; T19). Como ambiente orientado pela metacognição atribuímos aqueles em que a metacognição é inserida na atividade experimental para fomentar resultados como ganhos cognitivos, entre outros (T11; T15; T18).

Os resultados indicam que os pesquisadores que conduzem investigações sobre metacognição e atividades experimentais estão interessados em intervenções nas três possibilidades. No caso do ambiente natural, mencionamos o estudo de Kung e Linder (2007) - T5 que mostram, sem alterar a proposta de atividade, as manifestações metacognitivas presentes na fala de um grupo de estudantes. No estudo, as autoras assinalam que nessas manifestações espontâneas estão presentes as de natureza metacognitiva, todavia, apontam para a dificuldade em distinguir as manifestações cognitivas das metacognitivas, a exemplo do alertado por Flavell (1979).

Como exemplo de atividade experimental orientada para a metacognição, temos o artigo de van Optsal e Daubenmire (2015) - T14 que utilizou a Escrita Científica Heurística para desenvolver as habilidades metacognitivas na resolução de problemas abertos de laboratório. A investigação de Davidowitz e Rollnick (2003) - T2 também é guiada para a metacognição porque busca aprimorá-la com a utilização do tripé de 
competências e do diagrama de fluxo. No tripé de competências, o estudo realça fatores como o conhecimento declarativo, o conhecimento procedimental e a competência de comunicação, ligados por um anel e que têm sobre eles outros dois fatores importantes no decorrer das atividades experimentais: as interações humanas e o gerenciamento do tempo. O diagrama de fluxo, muito próximo a um mapa conceitual, foi entendido como uma representação esquemática da relação entre ideias e conceitos e era construído pelos estudantes antes de desenvolverem a atividade experimental.

Além desses, temos outros dois estudos vinculados a momentos explícitos da metacognição. O primeiro deles, desenvolvido por Saribaş et al., (2013), utiliza prompts metacognitivos para desenvolver ganhos conceituais, habilidades do método científico e visão da natureza da ciência. Os prompts são esquemas que os alunos usam como apoio ou guia para ativar o pensamento metacognitivo, normalmente elaborados com questionamentos gerais e não específicos do assunto em discussão. $\mathrm{O}$ segundo, referente à investigação de Bruckermann et al. (2017), analisa a influência do andaime metacognitivo na competência de experimentação. Os andaimes são ferramentas utilizadas como suporte para uma determinada atividade e que, com o passar do tempo e do uso, podem ser excluídos, pois os estudantes já conseguem realizar o procedimento sem o auxílio desse suporte.

Como terceiro aspecto investigado dentro dessa segunda categoria, apontamos a organização didático-metodológica das atividades experimentais das 13 pesquisas em que a metacognição ocupou papel de centralidade. A classificação tomou por referência as discussões apontadas por pesquisadores envolvidos com a temática das atividades experimentais, como os mencionados na introdução deste artigo. Buscamos avaliar nos 13 estudos selecionados o modelo de atividade experimental com o respectivo grau de abertura no caso das investigativas, elucidando se a atividade foi desenvolvida de forma individual ou em grupo e se o laboratório era real ou virtual.

Dos 13 estudos analisados, identificamos que 11 deles estavam associados a uma abordagem investigativa. Essas atividades investigativas estavam orientadas a buscar estimular a participação ativa do estudante e oportunizar a construção do conhecimento por meio da realização de investigações guiadas por questionamentos/perguntas. Esse tipo de abordagem inclui diferentes graus de liberdade em relação à ação do estudante, podendo ir das atividades mais fechadas com menor grau de liberdade (professor é responsável por definir o problema e os procedimentos), até a mais aberta em que há total liberdade para desenvolver a atividade (professor designa apenas o objeto de estudo).

Sobre esse grau de liberdade, que pode variar nas atividades experimentais investigativas, temos o estudo de Borges (2002) que apoiado em Tamir (2001) apresenta os graus de liberdade em níveis, assinalando do Nível zero (problemas, procedimento e conclusão são de responsabilidade do professor) até o Nível 3 (problemas, procedimentos e conclusão são abertos e ficam sob gerência dos estudantes). No Nível 1, o problema e os dados são de responsabilidade do professor e no Nível 2 somente a formulação do problema a ser investigado fica sob responsabilidade do professor. 
A pesquisa de Davidowitz e Rollnick (2003) - T2 desenvolvida em Química sobre o ciclo de cobre e o manganês no aço, partia de um problema definido pelo professor e os procedimentos estavam dados pelo manual de laboratório. Ao aluno couberam a geração, a interpretação e a comparação dos resultados com as especificações do fabricante. No artigo de Hand, Wallace e Yang (2004) - T3 foi usado o modelo tradicional de atividades experimentais para o grupo controle (CG) e o investigativo para os grupos que utilizaram a Escrita Científica Heurística (SG e STG). A Escrita Científica Heurística impele a adoção de uma abordagem centrada no estudante. A definição do procedimento e o resultado estavam sob a responsabilidade dos alunos, restando ao professor estabelecer o problema ou pergunta central. Foi adotada uma estratégia associada à atividade investigativa, na qual o professor auxiliava o estudante na construção do conhecimento. $\mathrm{Na}$ atividade tradicional, foi mantida a prática pedagógica utilizada pelo professor, na qual praticamente não havia controle algum dos alunos sobre as aulas.

A pesquisa desenvolvida por Kung e Linder (2007) - T5 foram utilizados três modelos de atividades experimentais: tradicional (dois grupos), tradicional acrescido de questões do tipo "explique seu raciocínio" nas instruções (dois grupos), e o investigativo (quatro grupos), onde os estudantes recebem a pergunta a ser investigada e, posteriormente, defendem seu método e resultados para os outros grupos. O processo atribuído aos estudantes foi o de determinar o procedimento adequado e produzir as conclusões, a partir de um problema vir pré-definido.

No estudo de Kipnis e Hofstein (2008) - T6 foi conduzida uma atividade experimental investigativa em Química envolvendo ácidos-bases, estequiometria, redução da oxidação, ligação química, energia, equilíbrio químico e taxa de reação. $\mathrm{Na}$ atividade o problema, o procedimento e a conclusão eram atribuições dos estudantes que tinham a oportunidade e o tempo necessário para discutir o fenômeno científico com os demais membros do grupo - momento de ativação do pensamento metacognitivo. A professora e o assistente de ensino orientaram e apoiaram a condução da investigação, por meio de discussões, fornecendo explicações quando solicitados.

As pesquisas desenvolvidas por Sandi-Urena, Cooper e Gatlin (2011) - T7, assim como por Sandi-Urena et al. (2011) - T8 e Sandi-Urena, Cooper e Stevens (2012) - T9, foram realizadas em pequenos grupos de estudantes que recebiam o problema a ser investigado e eram responsáveis pela definição dos procedimentos, condução do experimento e avaliação dos resultados. Ao final da atividade, os estudantes apresentaram suas decisões e conclusões para a turma e responderam às dúvidas dos colegas e do assistente de ensino.

No estudo de Saribaş, Mugaloğlu e Bayram (2013) - T11 houve um conjunto de experimentos sobre taxa de reação, equilíbrio químico, solubilidade, precipitação, ácidos e bases. Essas atividades foram realizadas por pequenos grupos de trabalhos (licenciandos em Química), divididos em grupo controle e grupo experimental. O primeiro grupo realizou atividades experimentais tradicionais, seguindo o manual de laboratório para encontrar respostas às perguntas nele apresentadas e, ao final, estruturar 
um relatório. O grupo experimental discutiu, antes e depois, os procedimentos do experimento, promovendo consciência metacognitiva. Os estudantes vinculados a esse grupo preencheram formulários reflexivos no início e no final das aulas e de cada experimento. Além de preparar o relatório final, o grupo experimental analisou e explicou implicações dos experimentos no cotidiano. Apesar de mencionar que as atividades investigativas deveriam engajar os alunos na identificação do problema, não fica claro se no estudo conduzido os estudantes ficaram responsáveis por essa etapa.

O estudo de van Optsal e Daubenmire (2015) - T14, semelhante ao anterior, utilizou dois modelos de atividades experimentais: investigativa e tradicional. A primeira foi realizada em grupos de três ou quatro alunos que discutiam questões para as investigações, os procedimentos e os resultados. Nesse modelo de atividade, o professor guiava as discussões com perguntas, encorajando a discussão entre pares. O modelo por eles denominado de "tradicional" foi desenvolvido em duplas (devido à referência ao parceiro de laboratório) e seguiu roteiro altamente estruturado, chamado de "manual de experimentos laboratoriais". O problema aberto apresentado após as atividades experimentais requeria dos alunos a determinação da pergunta, o planejamento, a condução e a avaliação do experimento.

Bruckermann et al. (2017) - T15, realizaram um estudo associado ao conceito de osmose envolvendo atividades experimentais investigativas realizadas em grupos de três a quatro alunos que conduziam a atividade, orientados por assistentes de ensino. A questão a ser investigada era a mesma de modo a permitir a comparação entre os quatro grupos. Os estudantes deveriam escolher o procedimento que considerassem mais adequado para resolver a tarefa. No final do experimento, o relatório feito pelos alunos para documentar o processo investigativo era apresentado e refletido com todos os colegas.

A pesquisa realizada por Mathabathe e Potgieter (2017) - T16 é a simulação de um projeto industrial que combinou elementos de investigação e colaboração entre pares para avaliar experimentalmente, qual das três formas de obter um composto orgânico apresenta melhor custo-benefício, é mais ecológica e tecnicamente menos desafiador. A fim de realizar a simulação, os universitários se reuniram em grupos de três pessoas, denominados "grupos base", onde cada um era responsável por avaliar uma das formas de obter o composto. Para gerar os procedimentos detalhados e conduzir o experimento, os estudantes dos grupos base se reuniram em "grupos de especialistas" que foram alocados na mesma rota sintética.

Em relação à atividade investigativa, percebemos que o problema era previamente estruturado, mas os procedimentos e as conclusões ficaram a critério do grupo de alunos que assumiram o papel de especialistas. Após concluir o experimento, os estudantes retornaram aos seus grupos base para auxiliar na recomendação de uma rota sintética baseada em decisões informadas. Ao professor e ao assistente de ensino coube esclarecer os requisitos da tarefa, responder às dúvidas estimulando a reflexão e garantir que fosse projetado um procedimento seguro. 
No estudo de Teichert et al., (2017) - T18, foi utilizado o modelo de atividades experimentais que os autores nomeiam de MORE, que é a sigla adotada para designar Model-Observe-Reflect-Explain. Em um artigo anterior, Rickey e Stacy (2000) explicam que as atividades experimentais "MORE" foram criadas a partir da POE (PredizerObservar-Explicitar), mas com diferenças fundamentais. Na MORE os alunos devem apresentar em detalhes suas ideias iniciais, refletindo sobre vários aspectos, em vez de fazer predições de resultados isolados. Além disso, é solicitada explicitamente a reflexão sobre as observações para refinar os modelos iniciais. Os estudantes relatam em palavras, figuras e símbolos o modelo inicial baseado em seus conhecimentos prévios sobre o tema - nesse caso, estrutura molecular de soluções aquosas. Após discutir com os colegas o modelo inicial, os estudantes conduzem experimentos, geram evidências macroscópicas diretamente relacionadas à estrutura molecular e são explicitamente solicitados a refletir sobre as implicações das observações para o modelo inicial. A partir das reflexões e usando evidências, os acadêmicos escreviam seu modelo final explicando como e por que as ideias iniciais foram modificadas ou não.

Acuthan et al., (2017) - T19 recorrem à associação do laboratório virtual com o físico (real) e vinculam a metacognição a essas atividades. A partir da leitura do artigo, não é possível dizer qual o modelo de atividades experimentais utilizadas no laboratório físico (real), mas identificamos que foi realizado em grupo. As atividades do laboratório virtual foram desenvolvidas individualmente para evitar que os alunos participassem passivamente das atividades em virtude da divisão de tarefas. O site (http://vlab.amrita. edu) utilizado para a condução dos experimentos inclui a teoria, os procedimentos, a simulação, entre outras informações.

Para dar continuidade à análise dos 13 estudos que apresentaram a metacognição como aspecto central, passamos a analisar o entendimento de metacognição neles presente. Nesse aspecto foram avaliados os artigos que se enquadraram como centrais, entretanto, no artigo de Acuthan et al., (2017) - T19, não foi possível identificar o entendimento de metacognição. Na leitura dos 12 artigos restantes, foi possível encontrar três entendimentos de metacognição que emergem do próprio entendimento de metacognição apresentado na introdução deste texto: a metacognição com enfoque nas ações regulatórias da cognição; a metacognição como um processo reflexivo; e, por fim, a metacognição abrangendo tomada de consciência e regulação da cognição.

Por regulação da cognição ou autorregulação entendemos o que na introdução foi especificado como controle executivo e autorregulador, referindo-se ao processo interno do estudante que controla a execução de uma determinada tarefa, envolvendo operações de planejamento, monitoração e avaliação. Essa compreensão está associada ao componente controle executivo e autorregulador, ou, como denominado por Veenman et al., (2006), às habilidades metacognitivas. Restringir o entendimento de metacognição a essa componente tem sido algo frequente nos estudos que envolvem a metacognição e os processos educativos, particularmente, a sala de aula, como aponta Veenman (2012), ao analisar como a metacognição foi integrada ao campo da Educação 
em Ciências. Tal entendimento pode ser percebido nos artigos de Sandi-Urena, Cooper e Gatlin (2011) - T7, Sandi-Urena et al. (2011) - T8, Sandi-Urena et al., (2012) - T9, van Optsal e Daubenmire (2015) - T14 e Bruckermann et al. (2017) - T15.

Kipnis e Hofstein (2008) - T6 acrescentam a esse entendimento expresso por Veenman (2012) o elemento controle e avaliam o aspecto social da metacognição (autorregulação e regulação de outros), trazendo para o estudo uma aproximação com os elementos metacognitivos associados à componente autorregulatória da ação cognitiva. Davidowitz e Rollnick (2003) - T2, por seu turno, buscavam compreender como os estudantes controlavam a situação de aprendizagem. Apoiados na definição de Gunstone (1994), os autores trazem que o aluno metacognitivo assume as tarefas de monitorar, integrar e ampliar seu próprio aprendizado, ou seja, possui bons comportamentos de aprendizagem, o que vem ao encontro da componente metacognitiva associada ao controle executivo e autorregulador. Em Kung e Linder (2007) - T5, a metacognição foi associada a julgamento e avaliação da cognição, como ressaltado por Georghiades (2004), todavia, o trabalho confere importância à componente de autorregular a ação como forma de revisão e julgamento dessa ação. Teichert et al. (2017) - T18, por sua vez, relatam que as atividades impulsionavam os estudantes a monitorar e regular seu próprio pensamento e aprendizagem, explicando como e por que revisaram seus modelos iniciais. Os pesquisadores não especificam autores que tratam da metacognição, todavia, seu entendimento traz a compreensão da metacognição como uma ação executiva e associada ao monitoramento e ao controle dessa ação. A metacognição, para Teichert et al. (2017), é utilizada para explicar como as evidências refutaram (ou confirmaram) os modelos iniciais, implicando no refinamento (ou não) do modelo final, e, como entendimento de metacognição, os autores apontam a presença das componentes autorregulatórias da ação.

É importante destacar que, embora essas pesquisas tenham focado na regulação do conhecimento, alguns autores reconhecem em seus artigos que a metacognição também abrange o conhecimento do conhecimento envolvendo a tomada de consciência (T7; T9; T14), ainda que não o utilizem diretamente nos estudos. No artigo de Mathabathe e Potgieter (2017) - T16, não foi mencionado explicitamente, mas há compreensão de que a componente do conhecimento do conhecimento é igualmente importante.

$\mathrm{O}$ entendimento de metacognição como um processo reflexivo, sem envolver detalhamento em componentes, foi uma primeira compreensão dada por Flavell, cujas raízes estão no pensamento reflexivo discutido pelo pedagogo americano John Dewey e que tem, em certa medida, prevalecido nos estudos. Essa compreensão foi questionada por Tarricone (2011), ao mencionar que, com o avanço das pesquisas, surgem detalhamentos dessa definição e que por vezes são ignorados pelos autores, permanecendo na superficialidade do conceito. Dentre os 13 estudos investigados, tal compreensão foi identificada em dois, que recorrem a expressões como pensamento sobre o próprio pensamento (Hand; Wallace; Yang, 2004) - T3 e consciência metacognitiva (Saribaş; Mugaloğlu; Bayram, 2013) - T11. Não são apresentadas mais informações sobre o que os autores compreendem como metacognição. 
No que se refere à compreensão da metacognição como tomada de consciência e monitoração do pensamento, temos como referência o apresentado inicialmente: a metacognição se apoia em duas componentes, uma ligada ao conhecimento que o sujeito tem sobre seu próprio conhecimento (consciência) e outra associada à regulação dada aos processos executivos (monitoramento). Vinculadas a essas duas componentes, estão as experiências que são desencadeadoras dos sentimentos em relação ao objetivo cognitivo a ser alcançado. Essa compreensão é discutida por Flavell et al., (1999), que, inclusive, compõem os referenciais teóricos adotados pelo único trabalho enquadrado nessa categoria. Kipnis e Hofstein (2008) - T6 apresentam uma discussão envolvendo o uso de atividades experimentais estruturadas a partir dessas duas componentes que no estudo são enaltecidas como tomada de consciência e monitoração do pensamento.

\section{Conclusão}

A pesquisa aqui relatada buscou responder à seguinte pergunta: quais são as características identificadas nas pesquisas estrangeiras que vinculam a metacognição com as atividades experimentais no ensino de Ciências? Para tanto, selecionamos 19 artigos utilizando a base de dados ERIC e verificamos em relação a um panorama geral das pesquisas que: a temática metacognição continua em crescimento, conforme haviam assinalado Zohar e Barzilai (2013); cinco revistas periódicas ocuparam um lugar de destaque visto que nessas publicações o somatório sobre essa temática correspondeu a cerca de $70 \%$ do corpus do estudo; não há uma distribuição uniforme dos estudos nos países, permitindo inferir que a importância dessa temática tem sido reconhecida em vários países e por diferentes pesquisadores; um número reduzido de estudos tem sido conduzidos na Educação Básica em contraste com um elevado número no Ensino Superior; e, ainda a Química foi o componente curricular com maior número de estudos.

Em relação à organização didático-metodológica, cinco investigações utilizaram mais de um tipo de atividade experimental para comparar os resultados do grupo experimental com o grupo controle, sendo utilizada a tradicional versus a investigativa em quatro delas. Dentre as doze pesquisas em que foi possível identificar a abordagem didático-metodológica das atividades experimentais, onze foram identificadas como investigativas (sendo investigativa sozinha ou utilizada para o grupo experimental). Dessas uma correspondeu ao Nível 1 de abertura, nove ao Nível 2 e uma ao Nível 3. Isso significa que os pesquisadores dos artigos que compõem o nosso corpus de estudo reconhecem que as atividades experimentais podem ter um nível de abertura intermediário, sem que a participação do aluno fique restrita a uma mera execução de roteiros estruturados ao mesmo tempo em que a responsabilidade conferida aos estudantes não se torne uma carga maior do que eles conseguem suportar. Em onze dos treze artigos em que a metacognição foi central, identificamos que as atividades experimentais foram conduzidas em grupo. $\mathrm{E}$ em nove artigos o professor não era considerado uma fonte de informações, mas sim um suporte que auxiliava os alunos a refletir e a encontrar as respostas para as suas dúvidas. 
Em termos de implicações da pesquisa para os estudos em metacognição e educação em Ciências, salientamos que ela apontou o entendimento dos autores de que as atividades experimentais investigativas se revelam uma estratégia potencialmente significativa em termos de ativação/evocação do pensamento metacognitivo. Entretanto, em uma análise mais detalhadas nos artigos selecionados para o estudo, identificamos uma carência em termos de discussões sobre o modo como ela é favorecedora dessa forma de pensamento. Embora tenhamos presente no discurso dos artigos, essa aproximação entre atividades experimentais investigativas e metacognição, os estudos se mostram tímidos em termos de evidenciar que aspectos da metacognição estão presentes e quais precisam ser explicitados durante a ação.

Por conta dessa identificação, percebemos que nas atividades experimentais analisadas a definição de metacognição utilizada foi, em geral, restrita à metacognição como um processo autorregulatório. A componente que se refere à tomada de consciência não aparece na forma de estratégias didáticas. $\mathrm{O}$ fato de essa componente não estar sendo operacionalizada significa que os autores estão atribuindo a tarefa de evocar os próprios conhecimentos aos estudantes. No entanto, o estudo de Santos et al., (2016) apontou que menos da metade dos alunos por elas investigados ativam espontaneamente essa forma de pensamento, principalmente os referentes à primeira componente da metacognição. A partir disso ponderamos que se espontaneamente a maior parte dos estudantes não recorre aos pensamentos metacognitivos, não seria sensato que as práticas os considerassem?

Tal inferência, como nos lembra Flavell (1979), apoia-se no entendimento de que a metacognição é resultante da integração das duas componentes, que não atuam de modo independente na estrutura cognitiva do sujeito, mas estão interligadas e constituem o conhecimento do conhecimento que o sujeito precisa ter para atingir seus objetivos de forma mais eficaz. Todavia, no momento de operacionalizar as propostas, $\mathrm{o}$ que tem ganhado relevância, como menciona Boszko (2019), são entendimentos como a reflexão de pensamento ou aspectos relacionados ao segundo componente anunciado por Flavell.

Após mapear as produções estrangeiras indexadas na base de dados ERIC e examinar os padrões contextuais e conceituais nelas emergentes, lembramos que o nosso corpus não abrange todos os artigos estrangeiros visto que podem existir estudos publicados em inglês que não foram indexados na ERIC assim como possivelmente existem estudos sobre essa temática publicados nas línguas maternas dos pesquisadores. Portanto, os resultados aqui apresentados e discutidos possuem essa limitação. Ainda assim, esses estudos indicam, ao menos em parte, o desenvolvimento da área. Como continuidade, o desejo está em nos debruçarmos sobre a análise de como as atividades experimentais investigativas podem se revelar promissoras da ativação do pensamento metacognitivo. 


\section{Referências}

Achuthan, K., Francis, S. P., \& Diwakar, S. (2017). Augmented reflective learning and knowledge retention perceived among students in classrooms involving virtual laboratories. Education and Information Technologies, 22(6), 2825-2855. https://doi. org/10.1007/s10639-017-9626-X

Bagán, H., Sayós, R., \& García, J. F. (2015). Skill development in experimental courses. Journal of Technology and Science Education, 5(3), 169-183. http://dx.doi.org/10.3926/ jotse. 158

Bomfim, A. B. C., Silva, S. A. P. S., \& Miranda, M. L. J. (2016). Knowledge production on continuous professional learning of physical education teachers: an analysis between brazilian and international studies. Journal of Physical Education, 27(1), e-2715. https:// doi.org/10.4025/jphyseduc.v27i1.2715

Borges, A. T. (2002). Novos rumos para o laboratório escolar de ciências. Caderno Brasileiro de Ensino de Física, 19(3), 291-313. https://periodicos.ufsc.br/index.php/ fisica/article/view/6607

Boszko, C. (2019). Diários de aprendizagem e os processos metacognitivos: estudo envolvendo professores de Física em formação inicial (Dissertação de Mestrado, Universidade de Passo Fundo, Passo Fundo, Rio Grande do Sul). Biblioteca de Teses e Dissertações - UPF. http://tede.upf.br:8080/jspui/handle/tede/1816

Bruckermann, T., Aschermann, E., Bresges, A., \& Schlüter, K. (2017). Metacognitive and multimedia support of experiments in inquiry learning for science teacher preparation. International Journal of Science Education, 39(6), 701-722. https://doi.org/10.1080/095 00693.2017.1301691

Çalişkan, Í. (2014). The Perceptions of Pre-Service Science Teachers' about Using Vee Diagrams and Electronic Portfolios in Physics Laboratuary Course. Education Research and Reviews, 9(6), 173-182. https://www.learntechlib.org/p/156652/

Carillo; L., Lee, C., \& Rickey, D. (2005). Enhancing Science Teaching by Doing More: A Framework to Guide Chemistry Students' Thinking in the Laboratory. The Science Teacher, 72(7), 60-64.

Carvalho, A. M. P. (2010). As Práticas experimentais no Ensino de Física. In A. M. P. Carvalho, Ensino de Física. Cengage Learning.

Clark, R. L., Clough, M. P., Berg, C. A. (2000). Modifying Cookbook Labs to Mentally Engage Students. The Science Teacher, 67(7), 40-43.

Davidowitz, B., \& Rollnick, M. (2003). Enabling metacognition in the laboratory: a case study of four second year University Chemistry Students. Research in Science Education, 33(1), 43-69. https://doi.org/10.1023/A:1023673122220 
Deslandes, S. F., Gomes, R., \& Minayo, M. C. S. (2012). Pesquisa social: teoria, método e criatividade ( $31^{\text {a }}$ ed.). Vozes.

Fadel, C., Bialik, M., \& Trilling, B. (2015). Four-dimensional education. Boston: Center for Curriculum Redesign.

Ferreira, A. V. (2012). A produção científica na educação ambiental: uma análise dos periódicos no banco de dados ERIC (Trabalho de Conclusão de Curso, Universidade Estadual de Maringá, Maringá, Paraná).

Flavell, J. H. (1979). Metacognition and cognitive monitoring: a new area of cognitive-developmental inquiry. American Psychologist, 34(10), 906-911. https://doi. org/10.1037/0003-066X.34.10.906

Flavell, J. H., Miller, P. H., \& Miller, S. A. (1999). Desenvolvimento cognitivo (3a ed.) [Cláudia Dornelles, Tradutora]. Artes Médicas Sul.

Galiazzi, M. C., Rocha, J. M. B., Schmitz, L. C., Souza, M. L., Giesta, S., \& Gonçalves, F. P. (2001). Objetivos das atividades experimentais no ensino médio: a pesquisa coletiva como modo de formação de professores de ciências. Ciência \& Educação, 7(2), 249-263. https://doi.org/10.1590/1516-731320200036

Ghani, I. B. A., Ibrahim, N. H., Yahaya, N., \& Surif, J. (2017). Enhancing students' HOTS in Laboratory Educational Activity by using Concept Map as an Alternative Assessment Tool. Chemistry Education Research and Practice, 18(4), 949-874. https:// doi.org/10.1039/C7RP00120G

Gil, A. C. (1994). Métodos e técnicas de pesquisa social (4ª ed.). Atlas.

Hacker, D. J. (1998). Definitions and empirical foundations. In J. Dunlosky, A. Graesser, Metacognition in educational theory and practice (pp. 1-23). Lawrence Erlbaum Associates.

Hand, B., Wallace, C. W., \& Yang, E-M. (2004). Using a Science Writing Heuristic to enhance learning outcomes from laboratory activities in seventh-grade science: quantitative and qualitative aspects. International Journal of Science Education, 26(2), 131-149. https://doi.org/10.1080/0950069032000070252

Hattie, J. (2012). Visible learning for teachers: maximizing impact on learning. Routledge. Heidemann, L. A. (2015). Ressignificação de atividades experimentais no ensino de Física por meio do enfoque no processo de modelagem científica. (Tese de Doutorado, Universidade Federal do Rio Grande do Sul, Porto Alegre, Rio Grande do Sul). LUME - UFRGS. http://hdl.handle.net/10183/117767

Hodson, D. (1988). Experimentos na ciência e no ensino de ciências [Paulo A. Porto, Tradutor]. Educational philosophy and theory, 20(1), 1-19.

Kipnis, M., \& Hofstein, A. (2008). The inquiry laboratory as a source for development of metacognitive skills. International Journal of Science and Mathematics Education, 6(3), 601-627. https://doi.org/10.1007/s10763-007-9066-y 
Kung, R. L., \& Linder, C. (2007). Metacognitive activity in the physics student laboratory: is increased metacognition necessarily better? Metacognition Learning, 2(1), 41-56. https://doi.org/10.1007/s11409-007-9006-9

Llorens-Molina, J. A., Jaime, J. M. L., \& Berzosa, I. S. (2012). Analysis of students' generated questions in laboratory learning environments. Journal of Technology and Science Education, 2(1), 46-55. http://dx.doi.org/10.3926/jotse.36

Luna, S. V. (2011). Planejamento de pesquisa: uma introdução. EDUC.

Malone, K. L. (2008). Correlations among knowledge structures, force concept inventory, and problem-solving behaviors. Physical Review Special Topics-Physics Education Research, 4(2), 1-15. https://doi.org/10.1103/PhysRevSTPER.4.020107

Marques, N. L. R. (2020). Formação e prática pedagógica: uma pesquisa sobre as contribuições das disciplinas de Física experimental para a prática docente na educação básica (Tese de Doutorado, Universidade Franciscana, Santa Maria, Rio Grande do Sul).

Mathabathe, K. C., \& Potgieter, M. (2017). Manifestations of metacognitive activity during the collaborative planning of chemistry practical investigations. International Journal of Science Education, 39(11), 1465-1484. https://doi.org/10.1080/09500693.20 17.1336808

Meneses, Jesús Á. (2018). Trabajos prácticos por indagación como estrategia para la enseñanza de la Física. In J. Á. Meneses Villagrá, \& M. J. F. Gebara (Orgs.), Estrategias didácticas para la enseñanza de la Física (pp. 121-142). Editora da Universidad de Burgos.

Nelson, T. O., \& Narens, L. (1994). Why investigate metacognition. In J. Metcalfe, \& A. P. Shimamura (Orgs.), Metacognition: knowing about knowing (pp. 1-25). ABB.

Pinho-Alves, J. (2000). Atividades experimentais: do método à prática construtivista. (Tese de Doutorado, Universidade Federal de Santa Catarina, Florianópolis, Santa Catarina). Repositório Institucional - UFSC. http://repositorio.ufsc.br/xmlui/ handle/123456789/79015

Rickey, D., \& Stacy, A. M. (2000). The role of metacognition in learning chemistry. Journal of chemical education, 77(7), 915-920. https://doi.org/10.1021/ed077p915

Romanowski, J. P. (2002). As licenciaturas no Brasil: um balanço das teses e dissertações dos anos 90 (Tese de Doutorado, Universidade de São Paulo, São Paulo, São Paulo). Biblioteca Digital de Teses e Dissertações da USP. https://doi.org/10.11606/T.48.2002. tde-22102014-134348

Rosa, C. T. W. (2011). A metacognição e as atividades experimentais no ensino de Física. (Tese de Doutorado, Universidade Federal de Santa Catarina, Florianópolis, Santa Catarina). Repositório Institucional - UFSC. http://repositorio.ufsc.br/xmlui/ handle/123456789/95261 
Rosa, C. T. W., \& Meneses Villagrá, J. A. (2018). Metacognição e ensino de Física: revisão de pesquisas associadas a intervenções didáticas. Revista Brasileira de Pesquisa em Educação em Ciências, 18(2), 581-608. https://doi.org/10.28976/1984-2686rbpec2018182581

Rosa, C. T. W., Darroz, L. M., \& Rosa, A. B. (2017). Discussões atuais e perspectivas futuras nas pesquisas brasileiras sobre metacognição e ensino de Física. Enseñanza de las ciências, Extra, 4245-4251.

Salvadego, W. N. C., Laburú, C. E., \& Barros, M. A. (23-26 de novembro, 2009). Uso de atividades experimentais pelo professor das Ciências Naturais no ensino médio: relação com o saber profissional. Anais do $1^{\circ}$ Congresso Paranaense de Educação em Química da Universidade Estadual de Londrina (CPEQUI), Londrina, Paraná.

Sandi-Urena, S., Cooper, M. M., \& Gatlin, T. A. (2011). Graduate teaching assistants' epistemological and metacognitive development. Chemistry Education Research and Practice, 12(1), 92-100. https://doi.org/10.1039/C1RP90012A

Sandi-Urena, S., Cooper, M. M., Gatlin, T. A., \& Bhattacharyya, G. (2011). Students' experience in a general chemistry cooperative problem based laboratory. Chemistry Education Research and Practice, 12(4), 434-442. https://doi.org/10.1039/C1RP90047A

Sandi-Urena, S., Cooper, M. M., Stevens, R. (2012). Effect of Cooperative Problem-Based Lab Instruction on Metacognition and Problem-Solving Skills. Journal of Chemical Education, 89(6), 700-706. https://doi.org/10.1021/ed1011844

Santos, A. C. T., Ribeiro, C. A. G., \& Rosa, C. T. W. (03-07 de outubro, 2016). Uso do pensamento metacognitivo por alunos do ensino médio. III Semana do Conhecimento, Passo Fundo, Rio Grande do Sul.

Santos, B. S. (2008). Um discurso sobre as ciências (5ª ed.). Cortez.

Saribaş, D., Mugaloğlu, E. Z., \& Bayram, H. (2013). Creating metacognitive awareness in the lab: outcomes for preservice science teachers. Eurasia Journal of Mathematics, Science \& Technology Education, 9(1), 83-88. https://doi.org/10.12973/eurasia.2013.918a

Sasseron, L. H. (2008). Alfabetização Científica no Ensino Fundamental: estrutura e indicadores deste processo em sala de aula (Tese de Doutorado, Universidade de São Paulo, São Paulo, São Paulo).

Taasoobshirazi, G., \& Farley, J. A. (2013). A Multivariate Model of Physics Problem Solving. Learning and Individual Differences, 24, 53-62. https://doi.org/10.1016/j. lindif.2012.05.001

Tarricone, P. (2011). The taxonomy of metacognition. Psychology Press.

Teichert, M. A., Tien, L. T., Dysleski, L., \& Rickey, D. (2017). Thinking Processes Associated with Undergraduate Chemistry Students' Success at Applying a MolecularLevel Model in a New Context. Journal of Chemical Education, 94(9), 1195-1208. https:// doi.org/10.1021/acs.jchemed.6b00762 
Van Optsal, M. T., \& Daubenmire, P. L. (2015). Extending Students' Practice of Metacognitive Regulation Skills with the Science Writing Heuristic. International Journal of Science Education, 37(7), 1089-1112. https://doi.org/10.1080/09500693.201 5.1019385

Veenman, M. V. (2012). Metacognition in science education: Definitions, constituents, and their intricate relation with cognition. In A. Zohar \& Y. J. Dori (Eds.), Metacognition in science education: trends in current research (pp. 21-36). Springer.

Veenman, M. V. J., Van Hout-Wolters, B. H. A. M., \& Afflerbach, P. (2006). Metacognition and learning: Conceptual and methodological considerations. Metacognition and Learning, 1(1), 3-14. https://doi.org/10.1007/s11409-006-6893-0

Zohar, A., \& Barzilai, S. (2013). A review of research on metacognition in science education: current and future directions. Studies in Science Education, 49(2), 121-169. https://doi.org/10.1080/03057267.2013.847261

Zômpero, A. F., \& Laburu, C.E. (2011). Atividades investigativas no ensino de ciências: aspectos históricos e diferentes abordagens, Revista Ensaio, 13(3), 67-80. https://doi. org/10.1590/1516-731320170020009

\section{Ana Claudia Tasso dos Santos}

Universidade de Passo Fundo Passo Fundo, Rio Grande do Sul, Brasil anatassosantos@gmail.com

Cleci Teresinha Werner da Rosa

Universidade de Passo Fundo Passo Fundo, Rio Grande do Sul, Brasil cwerner@upf.br

Editora Responsável

Marta Máximo

\footnotetext{
Manifestação de Atenção às Boas Práticas Científicas e de Isenção de Interesse

Os autores declaram ter cuidado de aspectos éticos ao longo do desenvolvimento da pesquisa e não ter qualquer interesse concorrente ou relações pessoais que possam ter influenciado o trabalho relatado no texto.
} 